\title{
On-line Monitoring Method of Bearing in Rotating Machinery Based on Wireless Sensor Networks
}

\author{
Nan Wang 1, 2, a, Yingxuan Liang ${ }^{1, b}$, Mingwu Wang ${ }^{1, c}$ \\ ${ }^{1}$ The School of Mechanical Engineering, Shaanxi University of Technology, \\ Hanzhong, PR China, 723001; \\ ${ }^{2}$ Shaanxi Key Laboratory of Industrial Automation, Shaanxi University of Technology, \\ Hanzhong, PR China, 723001; \\ a heroyoyu@126.com, b liangyingxuan@126.com, cwangmingwu@163.com;
}

\begin{abstract}
Keywords: Wireless sensor networks, On-line monitoring method, Routing protocol, Bearing of freight train, Water-lubricated bearing.

Abstract. For acquiring the precise parameters of bearing in rotating machinery, and further to study the bearing characteristics, and considering the difficult and bottleneck problems with wired monitoring method for bearing, the on-line monitoring method of bearing based on wireless sensor networks (WSNs) is proposed in this paper. Two typical engineering applications, namely the bearing of freight train and the water-lubricated bearing are studied. The key issues in application of WSNs, including the energy consumption, the routing protocol, the design of node, the compressive and coding algorithm of vibration data, and the Doppler effect are solved. Finally, the simulation and experiment results show the effective of the wireless method proposed.
\end{abstract}

\section{Introduction}

Bearing is the important foundational component of various mechanical equipments, because that it provides significant load capacity in order to support the rotor system, and the precision, lifetime and reliability of bearing play a decisive role for the performance of mechanical equipments. Rolling bearing and sliding bearing are the major categories of bearing in rotating machinery. According to the statistics, $30 \%$ of the faults in rotating machinery are caused by rolling bearings, and about one third of the world's energy consumption is currently consumed in different forms of friction, including one-tenth for sliding bearings [1]. So, the well carrying capacity, long lifetime and high reliability of bearing should be urgent required for modern rotating machinery, and the relevant on-line monitoring and fault prediction methods to rolling and sliding bearings are very important, which are also the key, difficult and hot issues for scholars all the time.

Off-line and on-line monitoring to bearing are the existing main methods. Because that the data obtained is discrete, and the early fault symptom and the overall process of fault occurrence unable be found, the off-line monitoring method is replaced by the on-line one [2]. Wire communication is adopted in most of the current on-line monitoring method, which is widely used and has many advantages, such as real-time capability, high efficiency, etc. But for bearings in rotating machinery, there are more kinds and structures about rolling and sliding bearings, which are applied to different types of mechanical equipments, and in addition, the application environment and industrial engineering of such bearings are major difference, so utilizing the on-line method for monitoring bearing has certain limitation in application, especially for the abominably industrial environment and some engineering application (cables not be easily used). The disadvantages of on-line wired monitoring method can be described as follows [3], (1) many cables and switches which are hard to be operated have potential safety hazard in some industrial site; (2)cables could not be permitted to be used, or hard to be used, or the data could not be transmitted by cables in some high-speed rotating equipments; (3)collector rings (commutator) used for the medium to transmit energy and data will bring signal drift, errors of measurement parameters or information, and so on; (4)signal attenuation and interference will be generated in long-distance transmission of analog signal. 
For solving the difficult and bottleneck problems with on-line wired monitoring method for bearing mentioned above, based on the excellent characteristic of wireless sensor networks (WSNs) (convenient and efficient installation, no wiring, self-organizing, data centered, etc), the monitoring scheme based on WSNs is proposed in this paper. Two typical engineering application objects, namely the bearing of freight train and the water-lubricated bearing are studied, the different topological structures and communication modes of network are adopted, the various key issues of each WSNs monitoring scheme (node design, energy consumption, the effect degree of high-speed mobile of equipment to the stabilization of node and network, etc) are solved, and the method proposed is proved to be effective by analyzing simulation and experimental results.

\section{Monitoring Method to bearing of Freight Train Based on WSNs}

Research Background. The freight train has many bearings, the structure and running modes are approximate linear, and in addition, the most important operating characteristics of freight train are unattended operation, hanging frequently, and the stabilized power could not be supplied. The bearing of freight train is rolling bearing (cylindrical bearing, conical bearing and self-aligning roller bearing), the monitoring parameters of bearing are noise obtained by ultrasonography monitoring or acoustic emission, temperature obtained by infrared or fiber monitoring, lubricating status obtained by infrared spectroscopy or ferrography, and vibration which is used much more widely. The monitoring method widely used for bearing of passenger train are on-board contact and rail-side induction detections, the monitoring equipments of rail-side induction detection include trace hotbox detection system (THDS) and trace acoustics detection system (TADS) [4, 5]. But because of some intractable technical problems, high cost in long-distance monitoring and operating characteristics of freight train, the leak detection and high misdiagnosis rate with bearing fault of freight train could not be solved satisfactorily by on-board contact and rail-slide induction detections.

Monitoring Scheme Based on Linear WSNs. Considering the approximate linear structure and running modes of freight train, the star network, cluster-shape network and mesh network are not applicable for monitoring, so the linear network and vibration of bearing are adopted for realizing the on-board contact mode [6,7]. There are various difficult problems which should be solved, such as routing protocol, node design, and processing algorithm for vibration data. Fig. 1 shows the monitoring scheme based on linear WSNs. There are two bogies, four wheel sets and eight bearings under each carriage of freight train, so two nodes are installed near the bearings under the carriage, and vibration signals with four bearings of a bogie could be collected by one node. The data and order information are transmitted among nodes by designed linear routing protocol, the base station arranged in train engine is used to receive the data and order information from other nodes, and finally, the data will be transmitted and processed in PC connected with base station. The node is battery powered, and the base station is powered by vehicle-mounted electrical power system. The characteristics of bearing vibration data are rapid changing, huge amounts and high real-time performance, and in addition, most of the energy of nodes and network are consumed in the process of data transmission, so in order to save the limited energy, the data acquired should be processed (compressed and coded) in node in advance, and the high efficient routing algorithm is also needed.

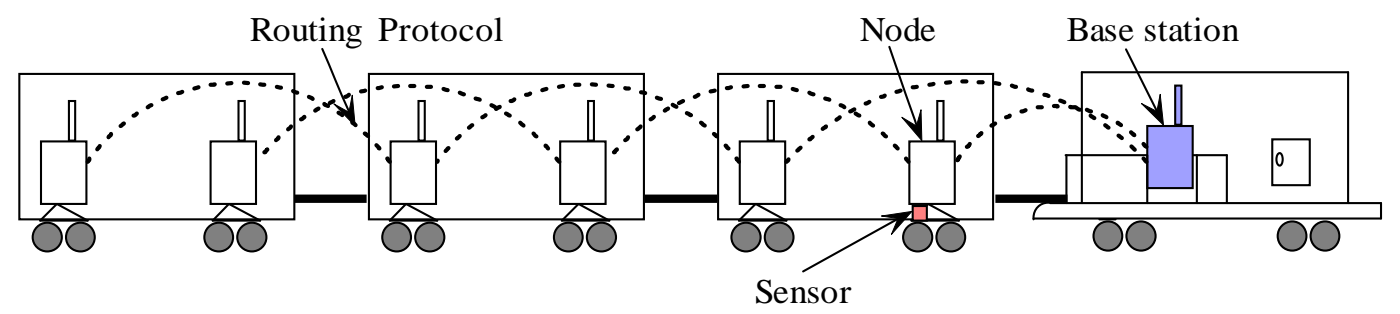

Fig. 1 Monitoring scheme for bearing of freight train based on linear WSNs

Linear Routing Protocol. The freight train consists of many carriages, and its structure and moving trajectory are straight line approximately, so the information transmission of node depends on relaying one by one between nodes. The structure and moving mode of freight train and information 
transmission mode easily lead to the imbalance of energy consumption between nodes, the lifetime of network will also be affected, therefore, the design of routing protocol (routing algorithm) is very important. The energy consumption of node depends on transmitting data size and transmitting distance of node, the functions of linear routing protocol are path planning for data transmission and balancing the energy consumption among nodes. Fig. 2 shows the grouped multi-hop routing based on equal distance (GMRED), there is no cluster head, the nodes of linear network are divided in to various groups, the nodes are uniform arrangement, and the node transmitting distance between groups is equal. This routing consists of single hop and multi hop, the construction and operation characteristics of freight train are considered, so the energy consumption of node in each group is equal, and the energy consumption of entire network is balance.

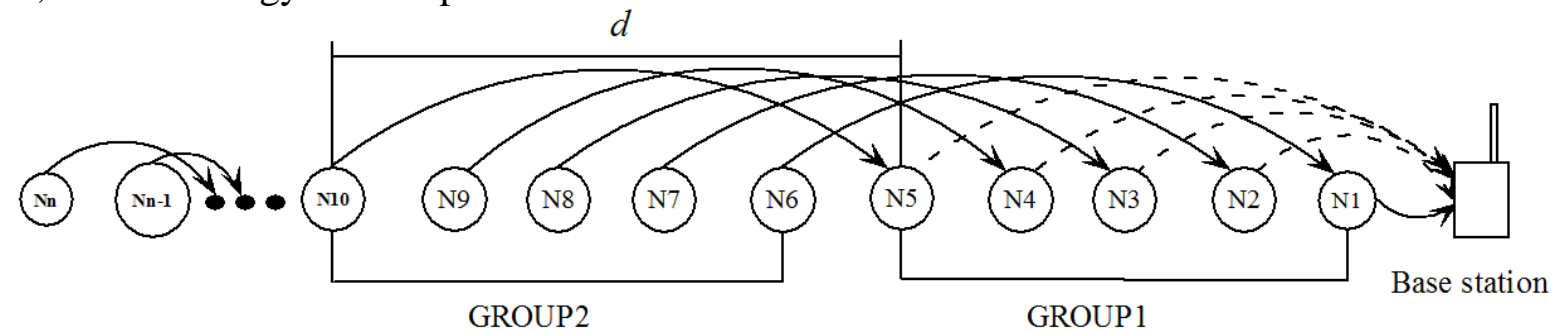

Fig. 2 Grouped multi-hop routing based on equal distance

Suppose that the same longitudinal data collected per node, the mathematical model of average energy consumption is obtained based on general network energy model [8], as shown in Eq. 1.

$$
\begin{aligned}
E_{a v e}=\frac{1}{N} \sum_{j=1}^{N_{e}} E_{g j}= & \frac{M\left[B\left(a_{r x}+a_{t x}\right)-2 c a_{r x}\right]}{2}+\frac{B \xi L^{2}}{6 N^{2}}\left[M+\frac{5 N^{2}}{M}-\frac{3 N^{2}}{M^{2}}\right] \\
& +\frac{2 N c a_{r x}\left(T_{d}+2\right)+N B\left(a_{t x}-a_{r x}\right)+B \xi L^{2}}{2 N}
\end{aligned}
$$

Where, $E_{g j}$ is node energy consumption, $N$ is amount of node, $M$ is amount of group $(1 \leq M \leq N)$, $B$ is data pakage lenth, $a_{r x}$ is the node energy consumption to receive a bit data, $a_{t x}$ is the node energy consumption to send a bit data, $c=0.2, \xi$ is the energy consumption of radio-frequency amplifier, $L$ is the length of network, $T_{d}$ is the period time. From Eq. 1 we can see that the average energy consumption $E_{\text {ave }}$ relates to the $L, N$, and $M$.

Fig. 3 shows the node energy consumption in a period of network using GMRED, while $M=20$, the energy consumptions of most nodes are in lower level. Fig.4 shows the node energy consumption in a period of network utilizing single-hop routing (SR), multi-hop routing (MR) and clustered multi-hop routing (CMR). From Fig.4 we can see that the energy consumption between each node is unbalanced by SR, MR and CMR, and comparing Fig.4 to Fig.3, the advantage of GMRED is obvious.

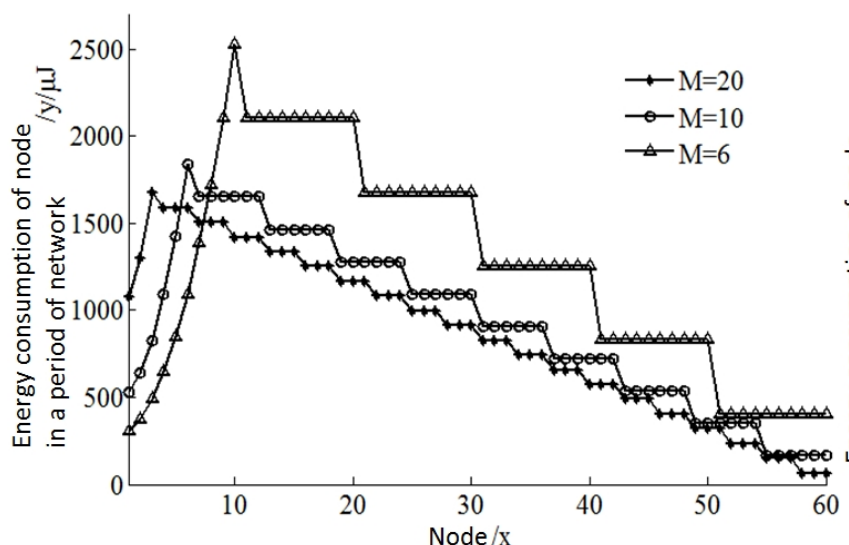

Fig. 3 Node energy consumption utilizing GMRED

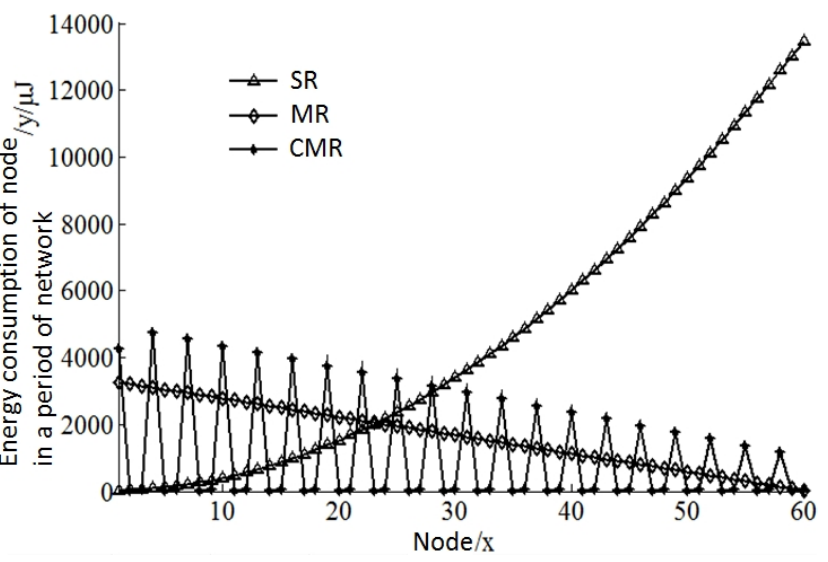

Fig. 4 Node energy consumption utilizing SR, MR and CMR

Node Design. The sensor node consists of sense module, processor module, RF sending and receiving module and power supply module, as shown in Fig. 5. The vibration sensor is powered by 
constant flow source, and a large number of vibration signals will be processed in digital signal processor (DSP). The parameter setting and routing judgment are conducted by single chip microcomputer (SCM), and the routing algorithm embed in RF chip is responsible for network establishment, routing and topology control. Fig. 6 shows the sensor node, four channel signals can be collected and processed simultaneously.

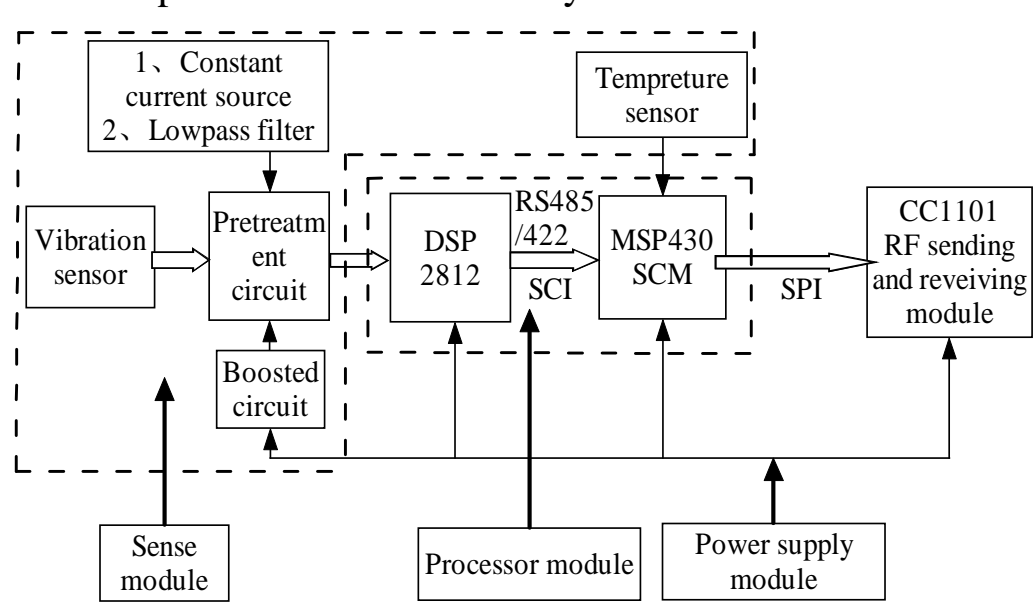

Fig. 5 Component of sensor node

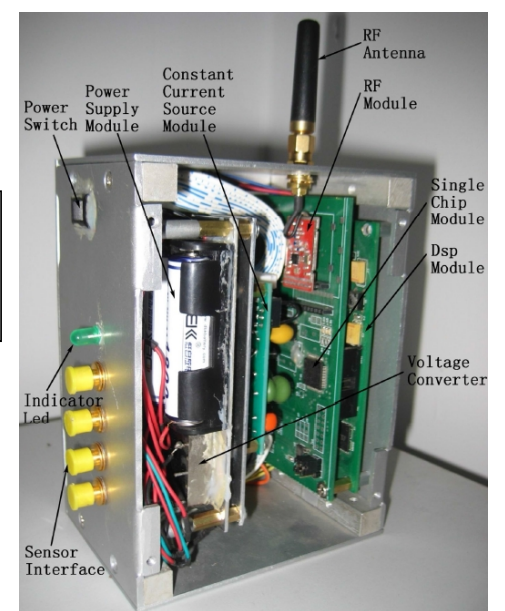

Fig. 6 Sensor node

Transmission scheme of vibration data. For reducing the data size of vibration signal, and further to save the limited energy of network, the compressive and coding algorithm is proposed and studied, as shown in Fig. 7. The acquired vibration signal from sensor is processed by $5 / 3$ lifting wavelet, embedded zerotree wavelet (EZW) [9] and Huffman code algorithm [10] separately in DSP of node, and then the processed data could be transmitted between nodes via wireless networks. The advantages of this algorithm are situ computation, high compressing efficiency, and easily realized in hardware.

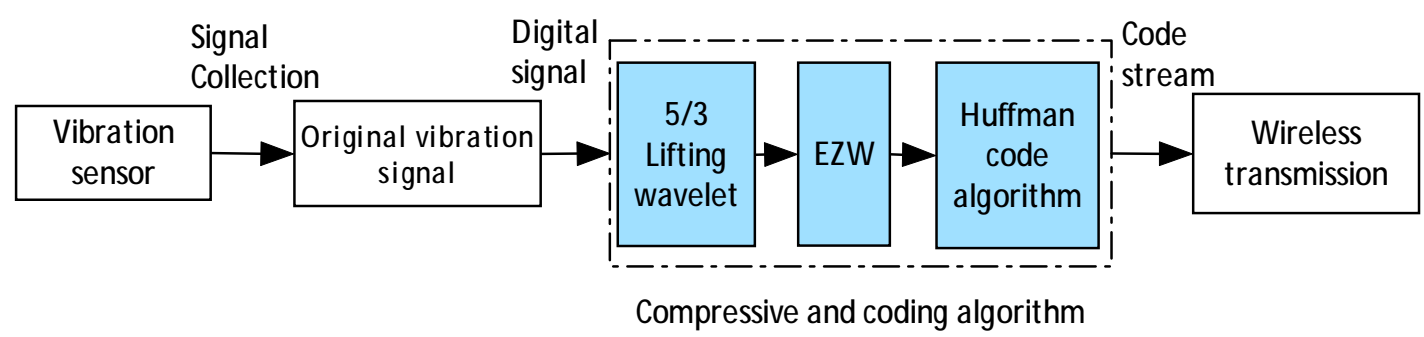

Fig. 7 Transmission scheme of vibration data

Fig. 8 shows the flow chart of compressive and coding algorithm, and the algorithm principle can be described as follows, in operational process of algorithm, wavelet decomposition retracts step by step, the calculated length of each level retracts $1 / 2$, and simultaneously, the threshold decreases to $1 / 2$. The main feature information of signal is in low frequency component sub-band, so the low threshold is used. In high frequency component sub-band, the non-feature information is filtered by algorithm with high threshold. In EZW, the important wavelet coefficients obtained by threshold in each level are stored in primary table, and the coefficient which is less than threshold is discarded. In the process of data compression above, the attached table which saves the position of important coefficients also produces. The function of attached table is to ensure the accuracy of data feature in the process of signal decompression. The construction of quantizer is not difficulty, and finally, the quantifying results are processed by Huffman coding algorithm, the code and data tables are produced. In decoded process, the data in data tables are decoded according to code table, and in decompressed process, the decoded data which are arranged according to attached table produced by EZW are reconstructed by lifting wavelet.

The compressing ratio relates to decomposition levels of wavelet, and initial threshold value, as shown in Fig. 9(a). The different decomposition level and initial threshold will greatly affect the compressive results, the calculating quantity increases as decomposition level increases, and this will lead to the increasing of CPU computational burden. The more decomposition levels of wavelet, the 
larger calculated quantity. The bigger initial threshold value selected, the larger compression ratio (CR) appeared. If the initial threshold changes greater, the wavelet coefficients during each scanning are less than before, then the compressive ratio increases, and the error of reconstitution signal increases. In Fig. 9 (b), the relationship between root mean square error ( $R M S E$ ) and wavelet level is unclear, when the wavelet level is 3, the fluctuation of $R M S E$ is larger than others. When level is 4, the fluctuation range of $R M S E$ is between that under 5 level and 6 level. The decomposition levels and initial threshold value should be set or selected according to actual application. By numerous tests, the CR of compressed and coded algorithm could reach up to 9.5 .

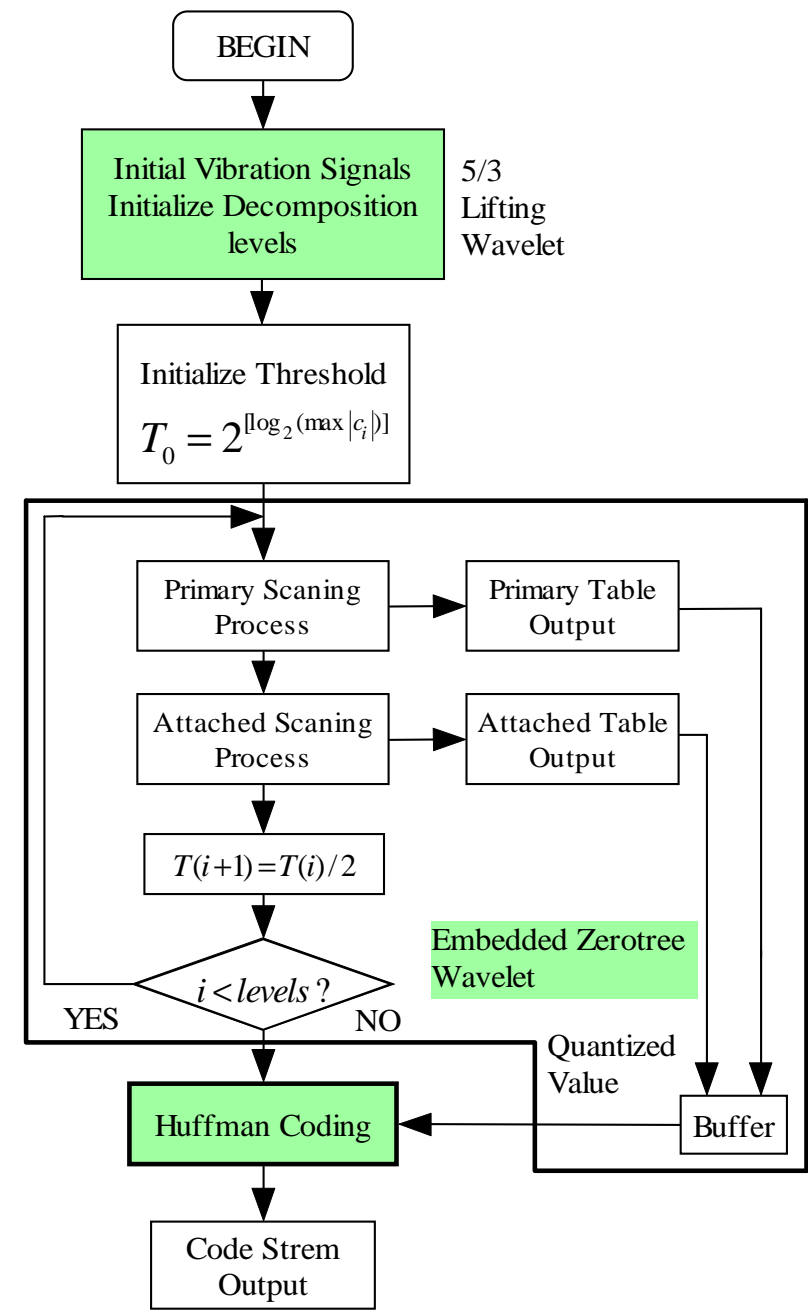

Fig. 8 Flow chart of compressive and coding algorithm

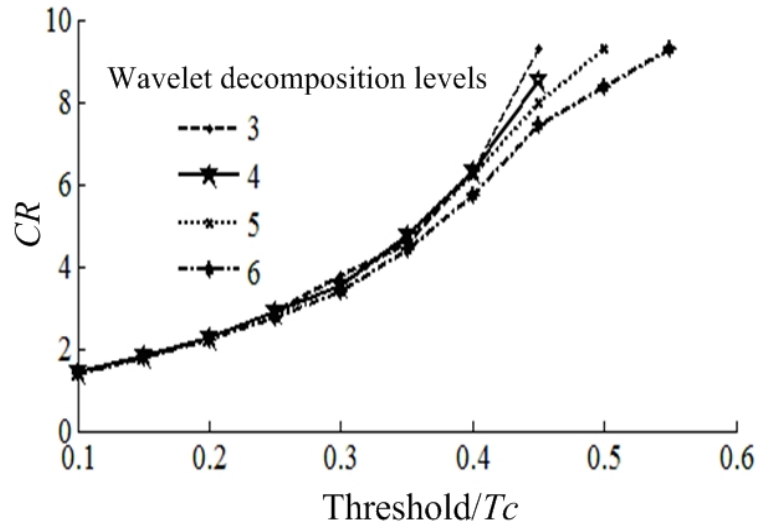

(a) $C R$ varies with $T c$

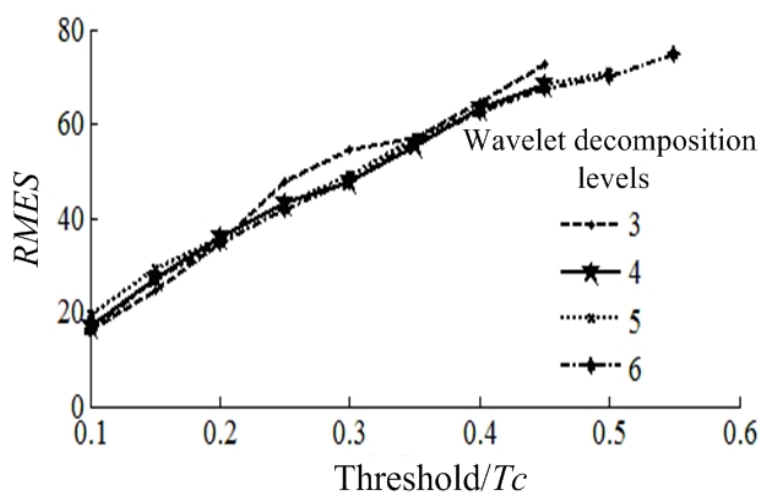

(b) RMES varies with $T c$

Fig. 9 Relationships among CR, RMES and threshold

\section{Wireless Testing Method of Water-Lubricated bearing}

Research Background. The water-lubricated bearing, which is one of the most appropriate bearings underwater, is used widely in ships, pumps, and others. The film pressure, which has received great attention for scholars, is the basic performance parameter of water-lubricated bearings. However, due to the restriction of many factors (sealing of film space, formation mechanism of film, and various bearing structures), the multi-points monitoring must be conducted, and the measurement for film pressure of bearings is hard to be achieved [11]. At present, there are two on-line monitoring methods, one method is that the pressure sensors are installed in the measuring holes punched on the shaft, and the data of film pressure is transmitted using the brush or mercury collection rings [12]. But the contact of ring produces more noises, which greatly affect the pressure, and furthermore, the reliability and the high cost of ring make its application limited. The other method is that the film pressure is measured by punching holes at different positions on bearing shell, leading the lubricant out to the pressure gauge or installing the pressure sensors in these holes [13]. Unfortunately, because 
of the measurement points on the bearing shell are limit, the continuous circumferential distribution of film pressure cannot be obtained. In addition, the two methods mentioned above are intrusive detection, the hole diameter contacting with lubricants is larger, and accordingly, the lubricating state of bearing working interface will be destroyed by testing point arrangement and sensor installation schemes.

Wireless Point-Point Monitoring Scheme. In order to obtain the continuous film pressure distribution of water-lubricated bearing, and further to study the characteristic and lubricating mechanism of bearing, as well as convenient to installation and debugging of sensors, the point-point monitoring method based on WSNs is proposed. Fig. 10 shows the wireless monitoring scheme for water-lubricated bearing, different from the existed method, the wireless monitoring method is noninvasive, the pressure sensors installed on end face of bearing connect with wireless equipment, and thus the film pressure signals are transmitted out of rotor system of bearing while the shaft revolves with high speed. There are two key issues need to be studied, one is the shaft design, the other is the information transmission quality affected by high speed movement of wireless equipment.

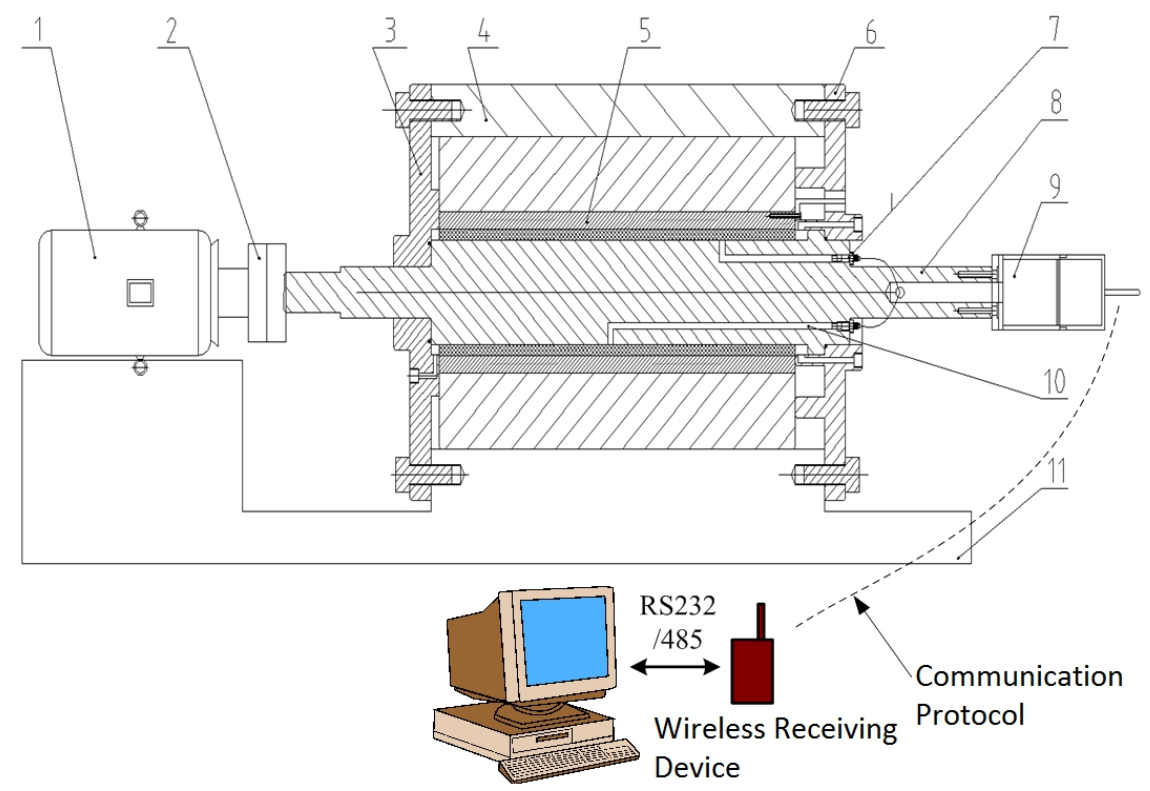

1-Motor; 2-Coupler; 3, 6-End cap; 4-Upper end cap; 5-Water-lubricated bearing; 7-Pressure sensor; 8-Shaft;

9-Wireless equipment; 10-Flow channel of lubricant; 11-Base of test-rig

Fig. 10 On-line wireless point-point monitoring scheme for water-lubricated bearing

Shaft Design. The arrangement of measurement point and the installation scheme of sensors are closely related to the design of shaft. Fig. 11 shows the structure of shaft, six radial flow guiding holes (pressure measurement points) are punched on the shaft along the axis with equal distance, and six axial flow guiding holes are punched on the right shaft shoulder. The axial holes, which are arranged in the circumferential direction with an angle of $60 \mathrm{deg}$, are connected with radial holes, and then the circumferential film pressure of the corresponding bearing sections could be propagated via the radial and axial holes. The film pressure sensors are installed in the axial flow guiding holes. By this design, the lubricating state of the bearing working face could not be damaged, the continuous circumferential film pressure can be acquired, and the installation and debugging of pressure sensors are convenient.

Doppler Effect. In this paper, the Doppler effect [14] is defined as, when the relative movement exists between transmitting device and receiving device, the phenomenon that the receiving frequency of information is different from the transmitting frequency of information. The difference between receiving frequency and transmitting frequency is called as Doppler frequency shift. The phenomena of bit error and packet loss of data while in the process of high speed rotation of wireless equipment, the influence factors of the phenomena, and its affect factor can be explained by Doppler effect in theory. The centring and balance of entire shafting is conducted before the test of water-lubricated bearing in advance, but because of the errors in the process of equipment manufacture, installation and alignment, the small circle oscillation $(0.04 \sim 0.5 \mathrm{~mm})$ of shafting 
appears, when the load changes from 0 to $1000 \mathrm{~N}$. Then this results in the relative movement between wireless equipments, and the Doppler frequency shift produces. The physical model of Doppler frequency shift is shown in Fig. 12, $D$ is diameter of swing circle $(\mathrm{m}), v$ is rotating speed of shaft (rpm, clockwise rotation), $\theta_{1}$ and $\theta_{2}$ are the angle of incidence of electromagnetic wave separately, $\Delta L$ is path difference of electromagnetic wave.

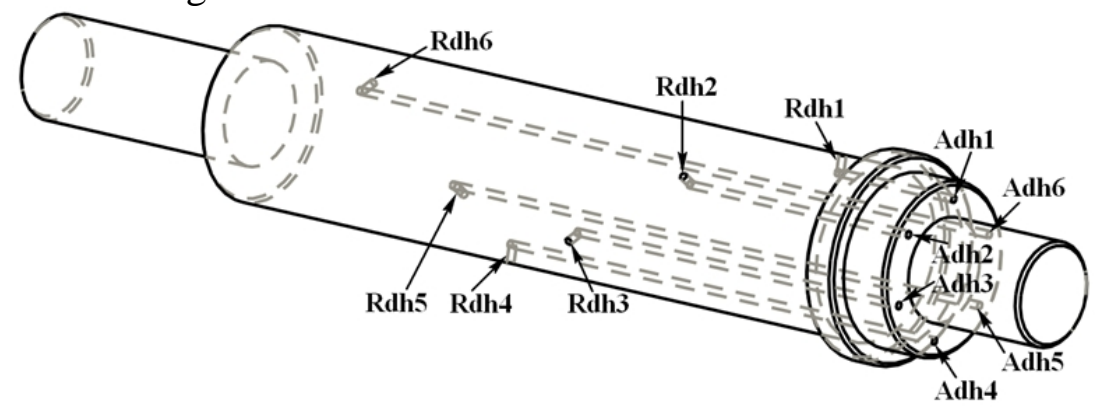

Rdh1 Rdh6: radial flow guiding hole; Adh1 Adh6: axial flow guiding hole

Fig. 11 Structure of shaft

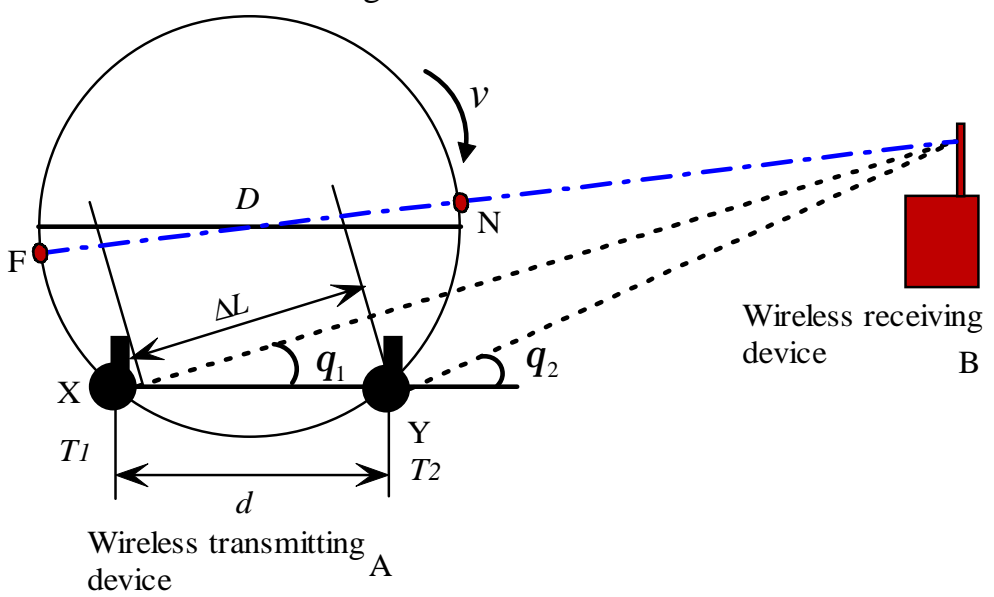

Fig. 12 Physical model of Doppler frequency shift

The mathematical model of Doppler frequency shift in theory is established, as shown in Eq. 2. Where, $f_{d}$ is Doppler frequency shift $(\mathrm{Hz}), \Delta \phi$ is phase change value of received signal, $\lambda$ is wave length of electromagnetic wave $(\mathrm{m}), f_{\max }$ is the max Doppler frequency shift $(\mathrm{Hz}), v_{d}$ is the relative rotating speed (rpm). See from Eq. $2, f_{d}$ relates to $D, v$, and $\theta$. In ideal conditions, $D$ is unchanged, $\theta$ has minor change, so $f_{d}$ mainly relates to shaft speed $(v)$. The larger of shaft speed, the larger of frequency shift, and accordingly, the frequency difference between receiving information and sending information with wireless equipments, thus, the bit error rate (BER) and packet loss rate (PLR) raise.

$$
f_{d}=\frac{1}{2 \pi} g \frac{\Delta \phi}{\Delta t}=\frac{D \sin (10800 \nu g \Delta t) / \Delta t}{\lambda} g \cos \theta=\frac{v_{d}}{\lambda} g \cos \theta=f_{\max } g \cos \theta
$$

\section{Conclusions}

In order to acquire the actual and precise data, and further to deeply study the characteristic of bearing, the on-line monitoring method based on WSNs is proposed. In monitoring on the bearing of freight train, the linear network and routing protocol are adopted. The GMRED routing algorithm is proposed for balancing the energy consumption of network, the average energy consumption $E_{\text {ave }}$ relates to the $L, N$, and $M$, and GMRED is proved to be effective by comparing to SR, MR and CMR. For compressing and coding algorithm, the different decomposition level and initial threshold will greatly affect the compressive results, the calculating quantity increases as decomposition level increases, and this will lead to the increasing of CPU computational burden. The compression ratio can reach up to 9.5 by conducting much more experiments.

In application of monitoring on the water-lubricated bearing, the point to point communication mode of WSNs is adopted, and the key issues are solved, including shaft design and Doppler effect. 
The arrangement of measurement point and the installation scheme of sensors are very important to conduct the wireless scheme, and which are decided by the design of shaft. The sensors installed have no contact with bearing lubrication interface, and the diameter of hole being contact with lubrication interface in shaft is only $1 \mathrm{~mm}$, so the destruction to lubricating state of bearing working interface decreases greatly, the measurement precision also improves. Finally, the information transmission quality affected by high speed movement of wireless equipment is studied, and by the analysis on model of Doppler effect, we found that, in ideal conditions, the Doppler frequency shift mainly relates to shaft speed.

\section{Acknowledgements}

This work was financially supported by the National Natural Science Foundation of China (51605269), the Projects Program of Shaanxi University of Technology Academician Workstation (fckt201510), and the Projects Program of Shaanxi University of Technology Doctoral Startup Fund (SLGQD 15-07).

\section{References}

[1] J. Zheng. Research on fatigue life of axle box bearing with high-speed train. Master thesis, Beijing Jiaotong University(2014) (in chinese).

[2] T.H. Yao, Y.L. Chi and Y.Y. Huang. Procedia Engineering, Vol. 31 (2012), p. 586-594.

[3] P.K. Upadhyay, L.A. Kumaraswamidhas and M.S. Azam. Case Studies in Engineering Failure Analysis, Vol. 1 (2013), p. 15-17.

[4] H.C. Choe, Y. Wan and A.K. Chan. Proceedings of SPIE - The International Society for Optical Engineering, Vol. 3078 (1997), p. 480-496.

[5] J.E. Cline, J.R. Bilodeau. Acoustic wayside identification of freight car roller bearing defects, Proceedings of the IEEE/ASME Joint Railroad Conference (1998), p. 79-83.

[6] A. Hossain, T. Radhika, S. Chakrabarti, et al. International Journal of Wireless Information Networks, Vol. 15 (2008), p. 72-81.

[7] L. Hong, S.J. Xu. Energy-efficient node placement in linear wireless sensor networks, International Conference on Measuring Technology and Mechatronics Automation, Changsha, China (2010), p. 104-107.

[8] M. Zimmerling, W. Dargie and J.M. Reason. Localized power-aware routing in linear wireless sensor networks, International workshop on context-awareness for self-managing systems, Sydney, NSW, Australia (2008), p. 24-33.

[9] J.M. Shapiro. IEEE Transactions on Signal Processing, Vol. 41, No. 12 (1993), p. 3445-3462.

[10] D.A. Huffman. Proceedings of the Institute of Radio Engineers, Vol. 40, No. 9 (1952), p. 1098-1101.

[11] R.S. Pai, R. Pai. Proc. IMechE Part J: J. Engineering Tribology, Vol. 222, No. J5 (2008), p. 683-691.

[12] D.L. Cabrera, N.H. Woolley and D.R. Allanson. Proceedings of the Institution of Mechanical Engineers, Part J: Journal of Engineering Tribology, Vol. 219 (2005), p.125-132.

[13] W. Litwin. Tribology Transactions, Vol. 54, No. 3 (2011), p.351-361.

[14] Y. K. An, S-M. Yoo, C. An, et al. Computer Communications, Vol. 36, No. 7 (2013), p. 834-848. 